\title{
Assembly rules of ectoparasite communities across scales: combining patterns of abiotic factors, host composition, geographic space, phylogeny and traits
}

\author{
Boris R. Krasnov, Georgy I. Shenbrot, Irina S. Khokhlova, Michal Stanko, Serge Morand \\ and David Mouillot
}

B. Krasnov (krasnov@bgu.ac.il) and G. Shenbrot, Mitrani Dept of Desert Ecology, Swiss Inst. Dryland Environmental and Energy Research, Jacob Blaustein Inst. Desert Research, Ben-Gurion Univ. Negev, Sede Boqer Campus, IL-84990 Midreshet Ben-Gurion, Israel. - I. Khokhlova, Wyler Dept Dryland Agriculture, French Associates Inst. Agriculture and Biotechnology of Drylands, Jacob Blaustein Inst. Desert Research, Ben-Gurion Univ. Negev, Sede Boqer Campus, IL-84990 Midreshet Ben-Gurion, Israel. - M. Stanko, Inst. Parasitology, Slovak Acad. Sci., Lofflerova 10, SK-04001 Kosice, Slovakia. - S. Morand, Inst. Sciences de l'Evolution, CNRS-IRD-UM2, CC65 and Animal et Gestion Intégrée des Risques CIRAD, Univ. Montpellier II, FR-34095 Montpellier, France. - D. Mouillot, UMR CNRS-UMII 5119 Ecosystemes Lagunaires, Univ. Montpellier II, CC093, FR-34095 Montpellier, France.

\begin{abstract}
We investigated the role of environmental filtering as an underlying mechanism of assembly of compound communities of fleas parasitic on Palearctic small mammals at two spatial scales; a continental scale (encompassing regions across the entire Palearctic) and a regional scale (across sampling localities within Slovakia). We used the three-table ordination (the RLQ analysis) and its extended version that links species occurrences with geographic space, environmental variables, and species traits and phylogeny (the ESLTP analysis). We asked whether environmental filtering acts as an assembly rule of compound communities of fleas and, if yes, a) whether the effect of environment on species composition of compound communities of fleas differs between spatial scales and b) what are the relative importance of the abiotic and host environments. We found that compound communities of fleas are, to a great extent, assembled via environmental filters that represent interplay between filtering via abiotic environment and filtering via host composition. The relative importance of these two components of environmental filtering differed between spatial scales. Host composition had a stronger effect on flea assembly than abiotic environment on the continental scale, while the opposite was true for the regional scale. The likely reason behind this scale-dependence is that communities on the regional scale are mainly governed by ecological and epidemiological processes, while communities on the continental scale are mainly affected by evolutionary, biogeographic and historical forces.
\end{abstract}

One of the central problems of modern community ecology is to understand the rules determining how species assemblies are formed at different scales (Chesson 2000). This task is not trivial since species composition within any community is affected by a variety of forces that act during both ecological and evolutionary times. Attempts to explain why a particular community comprises a given set of species have led to three main concepts underpinning assembly rules. First, the principle of limiting similarity among co-existing species may shape community composition (MacArthur and Levins 1967). Indeed, based on Darwin's (1859) idea that the intensity of competition is expected to increase with increasing biological similarity among competing species, species can only coexist if their overlap in resource use is limited because of morphological, ecological or behavioral dissimilarities (Hutchinson 1959, Schoener 1974, Kingston et al. 2000, Schluter 2000, Violle et al. 2011, Vergnon et al. 2013). Second, the environment may act as a 'filter', allowing a community to contain only species possessing certain adaptive traits that are necessary for persistence in that environment (Cavender-Bares et al. 2004, Ackerly and Cornwell 2007, Ingram and Shurin 2009). Finally, the 'neutrality' concept (Hubbell 2001) suggests that communities are primarily shaped by dispersal limitations and population dynamics since species belonging to the same trophic level are equal in their fitness and competitive ability. However, a pattern superficially resembling neutrality could arise due to the counter-balancing of competition and environmental filtering (Purves and Pacala 2005). Moreover the different mechanisms may operate simultaneously, but with different magnitudes of influence at different scales (Ingram and Shurin 2009, Weiher et al. 2011). Therefore, to better understand the structure of communities and predict their future in a very uncertain world the important ecological question may not be to identify an exact mechanism shaping ecological communities, but rather to determine the relative influence of each at a given scale. 
Recent developments of analytic techniques have allowed the application of phylogenetic tools to species composition data in order to reveal clear-cut mechanisms behind species composition in a variety of plant and animal communities (reviewed by Webb et al. 2002, Cavender-Bares et al. 2009, Pavoine et al. 2009, Weiher et al. 2011). In other words, investigation of assembly rules combined with modern phylogenetic methods allows disentangling the main drivers of structuring of ecological communities.

For the most part, communities of parasitic organisms have been neglected in studies that took into account both ecological and phylogenetic information despite the importance of parasites from both theoretical and applied perspectives. However, some attempts to link phylogeny of parasites and their community ecology have been made (Mouillot et al. 2006, Krasnov et al. 2014). The most conspicuous feature of the spatial distribution of parasites is that it is not continuous, but is fragmented among sets of inhabited 'islands' represented by individuals, populations and communities of their hosts. The commonly accepted hierarchical terminology in parasitology distinguishes between parasite communities on or in individual hosts (infracommunities), populations of conspecific hosts (component communities) and local communities of heterospecific hosts (compound communities) (Holmes and Price 1986, Poulin 2007). Obviously, parasite communities at these different levels differ sharply in, for example, time of persistence and the probability of individual parasites to interact with their conspecifics. It is thus not surprising that some rules governing these communities are dramatically different (Šimková et al. 2001) although other rules appear to be similar (Krasnov et al. 2006a versus Krasnov et al. 2011a). This suggests that an investigation of parasite communities at only one of the three hierarchical levels is a necessary but not sufficient condition for elucidating forces shaping parasite communities. Recently, we showed that environmental filtering rather than limiting similarity is a likely mechanism behind species composition of infracommunities and component communities of fleas parasitic on small mammals (Krasnov et al. 2014). Yet, it is still unknown whether compound flea communities follow the same rules.

Furthermore, contrary to free-living species, parasites are characterized by a 'dual' environment. On the one hand, this environment is represented by their hosts that provide parasites not only with food but also with a place for living. On the other hand, this environment is represented by abiotic variables. This is especially true for ectoparasites that are strongly affected by off-host environment. Environmental filtering may thus act on compound communities of parasites either as an 'abiotic environment filter' or a 'host environment filter'. In other words, parasite species in a specific locality may be filtered by factors such as air temperature or precipitation, and the occurrence of hosts belonging to a certain taxon or phylogenetic lineage. The relative importance of abiotic environment-related or host-related factors for the composition of parasite communities is largely unknown. In addition, community assembly rules may differ at different spatial scales (Weiher et al. 2011) since they are dependent mainly on factors acting during ecological time, on smaller scales, or on factors acting over evolutionary time on larger scales (Gonzáles and Oliva 2009, Krasnov et al. 2011b).
Here, we investigated the role of environmental filtering as an assembly mechanism of compound communities of fleas parasitic on small Palearctic mammals. We studied compound communities of fleas on two spatial scales, namely a) a continental scale, that is across the large part of the Palearctic and b) a regional scale, that is across sampling sites within a single region. Fleas are characteristic parasites of small and medium-sized mammalian species. They are holometabolous insects, and all species are obligate haematophages as imagoes, while the larvae of almost all species are non-parasitic and omnivorous. At both stages, fleas are strongly affected by abiotic, off-host environment (reviewed by Krasnov 2008), while feeding and reproductive performance of imaginal fleas depends strongly on host identity and phylogenetic position (Khokhlova et al. 2012). Flea species vary in the degree of their host specificity ranging from strictly host-specific to highly host-opportunistic (reviewed by Krasnov 2008). Our main question was whether environmental filtering acts as an assembly mechanism for compound communities of fleas, as it does for infra- and component communities of these parasites (see above). Assuming a positive answer to this question, we also asked a) whether the effect of environment on species composition of compound communities of fleas differs among spatial scales, and b) what is the relative importance of abiotic versus host environment as an assembly mechanism for compound communities of fleas at the continental and the regional scales.

To answer these questions, we took advantage of recent developments of three-table ordination techniques (the RLQ analysis; Dolédec et al. 1996) and its extended version (Pavoine et al. 2011). Traditional RLQ analysis allows one to link environmental variables at a set of sites with traits of species inhabiting these sites through species occurrences at these sites (see Material and methods for details). The extended version of the RLQ analysis (the ESLPT analysis) is a novel integrative methodology that allows analyzing an association between environment, species occurrences and species traits in an unambiguous geographic and phylogenetic context. In other words, this approach links species occurrences with geographic space, environmental variables, species traits and phylogeny to unravel complex assembly rules.

\section{Material and methods}

\section{Flea and host species composition}

On a continental scale, we obtained data on flea species composition in different regions from published surveys that reported the number of fleas of a given species found on a given number of individuals of a given species of a small mammal (Erinaceomorpha, Soricomorpha, Rodentia and Lagomorpha) in the northern and temperate Palearctic. Obviously, estimates of species composition of a parasite assemblage harbored by a host assemblage in a region can be reliable only if species compositions of parasite assemblages harbored by individual host species are accurately estimated for a majority of host species. Estimation of species composition of a parasite assemblage harbored by a given host species in a given region, in turn, can be inaccurate for small samples 
(Poulin 2007). Consequently, we included in the analyses only surveys that aimed to study the entire flea assemblage of the entire assemblage of small mammalian hosts in a defined region, while omitting studies aimed to investigate flea community harbored by a single target species. Then, we selected studied in which a) at least 10 individuals of each host species were examined for fleas, b) at least 500 individual mammals belonging to all host species were sampled and c) at least eight mammal species were found to harbor fleas. Parasitological examination of 10 conspecific individuals has been found to allow reliable estimation of flea species richness (Krasnov et al. 2005). In total, we carried out the analyses for 45 regions (see the list of the regions, map and references in Supplementary material Appendix 1). Then, we omitted those host species and their specific fleas, which required special sampling techniques and were sampled in part of the regions only (squirrels, flying squirrels, hedgehogs, and hares). A majority of the remaining host species were sampled using snap-traps and pitfall traps, while pikas, ground squirrels and marmots were hunted. In each region and for each host species, flea collecting technique was the same (visual examination and collecting all fleas from a dead animal immediately after capture). The selection procedure and the across-studies similarity of the field methods guaranteed comparability of the data on flea species composition among conspecific hosts across regions and among different hosts within a region. In total, for the continental scale analysis, we used data on 202 flea species collected from 561805 individuals belonging to $136 \mathrm{mammal}$ species.

For the regional scale analysis, we used data on fleas collected from small mammals in 13 localities across Slovakia (see map in Supplementary material Appendix 2). Small mammals were sampled using snap-traps during both the warm (May-September) and cold (OctoberApril) seasons. Sampling procedures and methods for parasitological examination were the same as above and details are given elsewhere (Stanko 1994). In total, for the regional scale we used data on 27 flea species collected from 13966 individuals belonging to 24 mammal species.

Regions across the Palearctic and sampling localities across Slovakia differed in area. Prior to our main analyses, we tested whether these differences affected the composition of recorded flea and host species using Mantel tests of correlation between two distance matrices, a matrix of pairwise dissimilarities between regions or localities in flea or host species composition versus a matrix of pairwise dissimilarities in area between regions or localities, with 999 replicates. We used the Rao dissimilarity index (Rao 1982) for the former(s) and the Mahalanobis distance for the latter. No correlation between species composition and sampling area was found for either fleas or hosts for either the continental or the regional scale (Mantel's $r=0.11-0.15$, $\mathrm{p}>0.12$ for all).

\section{Flea species properties (traits)}

No data on any measurable morphological or physiological traits (i.e. life-history) are available for the absolute majority of flea species used in this study. Consequently, in this study we considered as traits those properties of flea species that a) have been shown to be true attributes of a flea species and b) are highly ecologically important. Further, we refer to these properties as traits to be consistent with terminology accepted in the studies that used the RLQ or ELSTP analyses. We used six properties (traits) to characterize each flea species, namely a) preference to spending most of the time either on the body of a host or in its burrow or nest, or both; b) season of reproduction; c) mean characteristic abundance (that is, mean number of fleas per individual host); d) mean size of the host spectrum (that is, mean number of host species on which a given flea species was recorded); e) mean phylogenetic distinctness (PDH) of the regional host spectrum (see explanation in Supplementary material Appendix 3); and f) mean $\mathrm{PDH}$ of the continental host spectrum. Traits a) and b) (i.e. microhabitat preference and seasonality) were thus represented by sets of three binary variables each (Supplementary material Appendix 3). The remaining traits were quantitative. Traits d) to $\mathrm{f}$ ) represent different facets of host specificity. See Supplementary material Appendix 3 for the rationale and detailed explanations for the selection of flea traits and calculation of host specificity indices.

\section{Flea phylogeny}

The phylogenetic tree of fleas was based on the only available molecular phylogeny (Whiting et al. 2008). The positions of flea species that were not represented in the original tree of Whiting et al. (2008) were determined according to their morphologically-derived taxonomic affinities (for further details see Krasnov et al. 2011b). All branch lengths were arbitrarily set to an equal length of 1 and the tree were arbitrarily ultrametricized using the program Mesquite 2.75 (Maddison and Maddison 2011). The phylogenetic tree used in this study is presented in Supplementary material Appendix 4.

\section{Spatial, abiotic and host environment data}

We determined sampling area for a region or a locality either using data from an original source or by constructing a minimal convex polygon from the coordinates of sampling sites. Coordinates of the center of a region or locality were used as spatial data for subsequent analyses. We characterized the abiotic environment of each region or locality using topography (mean, maximum and minimum altitude), green vegetation [normalized difference vegetation indices (NVDI) for winter, spring, summer and autumn] and climatic variables (mean winter, spring, summer and autumn precipitation; mean, maximum and minimum air temperatures, annual and monthly temperature ranges). These variables were averaged within the area of a given region or locality across 30 arc-second grids (approximately $1 \mathrm{~km}$ resolution). Elevation data were extracted from the GOTOPO30 data set issued with ArcGIS Desktop 9.2. Data on green vegetation (NDVI) were obtained from the VEGETATION programme (<www.spot-vegetation.com>; <http://free. vgt.vito.be >; data for 1998-2007, estimates every 10 d). 
NDVI is a measure of photosynthetic activity on the landscape scale. Averaging of NDVI values across large areas provides a general estimate of green vegetation abundance (Pettorelli et al. 2005). Climatic variables were obtained as a part of WORLDCLIM ver. 1.4 (BIOCLIM) package (Hijmans et al. 2005). Further details can be found in Krasnov et al. (2010). Environmental variables were log-transformed prior to analysis. On both the continental and regional scales, we found significant correlation between the majority of topographic variables, vegetation and climatic environmental variables $(r=0.59-0.89$, $\mathrm{p}<0.005$ for all). Consequently, in further analyses we retained eight abiotic variables for the continental scale (minimum and maximum altitude, summer and winter NDVI, summer and winter precipitation, annual air temperature range, and maximum air temperature) and seven abiotic variables for the regional scale (maximum altitude, spring and summer NDVI, spring, summer and winter precipitation, and minimum air temperature).

To characterize a pool of host species that can support fleas in each region/locality (i.e. all host species on which at least one flea species was found) from both numerical and phylogenetic points of view, we used the number of host species belonging to one of 26 main phylogenetic/ecological clades/ groups (based on the host phylogenetic tree described above; see Supplementary material Appendix 5). These variables were considered ordinal. The host variables did not correlate significantly with each other (Spearman's ranks correlation coefficient $=-0.28-0.36, \mathrm{p}>0.05$ for all). Neither did the host variables correlate significantly with any abiotic variable (Spearman's rank correlation coefficient $=-0.20-0.33$, $\mathrm{p}>0.05$ for all).

\section{Analytical approach}

The traditional RLQ approach (Dolédec et al. 1996) is a three-table ordination. It considers species assemblages across sites and explores the relationships between environmental variables at these sites (matrix R; sites as rows and environmental variables as columns) and traits of species inhabiting these sites (matrix Q; species as rows and traits as columns) linking them via species occurrences at these sites (matrix L; sites as rows and species abundances or occurrences as columns). The RLQ reveals combinations of traits that strongly co-vary with combinations of environmental variables. One of the advantages of the RLQ analysis is its low sensitivity to the number of input variables as compared to other ordination methods (Dray et al. 2003). Recently, Pavoine et al. (2011) developed a novel, extended version of the RLQ analysis that allows to associate environmental variables (matrix E) with geographic space (matrix S) and trait variables (matrix T) with phylogeny (matrix P). In the extended RLQ analysis, referred to as the ESLTP analysis below, spatial variables together with environmental variables are introduced as columns of the matrix $R$, whereas phylogenetic variables together with trait variables are introduced as columns of the matrix Q. See Supplementary material Appendix 6 for the details of the strategy of the ESLTP analysis.

\section{Construction of matrices and selection of variables}

For each scale, we compiled five matrices (E, S, L, T, and $\mathrm{P})$. The matrix $\mathrm{L}$ was the matrix of presence or absence of each flea species in each region (for the continental scale) or site (for the regional scale), where a row represented a region or a locality, respectively, and a column represented a flea species. The matrix $\mathrm{E}$ included both quantitative variables describing the abiotic environment (further referred to as abiotic variables) and ordinal variables describing the host composition (further referred to as host variables). We tested for correlation among the environmental variables of each group (relief, vegetation and climate). We took into the analyses only those variables from each group that were not correlated with other variables or that had the lowest, albeit significant, correlation coefficient with other variables in a group. Prior to the ESLTP analysis, environmental variables have to be tested for spatial autocorrelation (Pavoine et al. 2011). The lack of spatial autocorrelation in an environmental variable indicates that it is not affected by space and thus should be omitted from the ESLTP analysis. We tested for spatial autocorrelation of both abiotic and host variables using a Moran's $I$ test (Thioulouse et al. 1995). Trait variables have to be tested for phylogenetic signal and correlation with environmental variables (Pavoine et al. 2011). A trait variable should be omitted from the ESLTP analysis if it does not demonstrate phylogenetic signal or does not correlate with environmental variables. To detect phylogenetic signal, we used the root-skewness test of Pavoine et al. (2010). To test for correlation of a trait with environmental variables, we used the multivariate version of the fourthcorner algorithm (Dray and Legendre 2008) and applied the null model 4 (permutations of the rows of species $\times$ trait matrix) (see Pavoine et al. 2011 for details). In addition, we tested whether flea species that co-occur in a region or a locality were significantly more or less a) phylogenetically related and b) similar in their traits than expected by chance from the continental or the regional, respectively, pool of species (phylogenetic or trait clustering versus phylogenetic or trait overdispersion; Webb et al. 2002, Pavoine et al. 2010). This was done using the PQE and TQE tests, respectively. These tests developed by Pavoine et al. (2010) and based on Hardy and Senterre (2007) are designed to evaluate the degree of environmental filtering versus limiting similarity.

As a result of selection of environmental and trait variables described above and because no significant autocorrelation in any environmental variable was found for the regional scale (see Results), we retained for the analyses on the continental scale only those environmental variables that demonstrated significant spatial autocorrelation, while all environmental variables were taken into the analyses on the regional scale (with the matrix $\mathrm{E}$ being the matrix $\mathrm{R}$ ). The analyses on the continental scale included traits that were characterized by significant phylogenetic signal and were significantly correlated with environmental variables. No significant phylogenetic signal was found for any trait on the regional scale (see Results), so the analyses on this scale included only traits significantly correlated with environment and the matrix $\mathrm{T}$ was used as the matrix Q. In other words, we did not use matrices $S$ and $P$ for the analyses on the regional scale. Thus, we analyzed data on the continental scale using the ESLTP 
analysis, whereas data on the regional scale were analyzed using the regular RLQ analyses.

\section{The ESLTP and the RLQ analyses}

Prior to doing the main ESLTP and the regular RLQ analyses, each original matrix was analyzed by a separate factorial analysis (= ordination). Details of these factorial analyses are presented in Supplementary material Appendix 7. Significance of the connection between the matrices $\mathrm{R}$ and Q (for both continental and regional scale) as well as between the matrices $\mathrm{E}$ and T, S and T, E and P, and S and P was tested with the multivariate version of the fourth-corner approach using null model 4 of Dray and Legendre (2008) (fixed environmental and/or spatial variables and randomly distributed species) (see Pavoine et al. 2011 for details).

At each scale, we carried out three versions of either the ESLTP (for the continental scale) or the regular RLQ (for the regional scale). In the first version, the matrix $\mathrm{E}$ (continental) or the matrix $\mathrm{R}$ (regional) contained information on both abiotic and host variables. In the second version, the matrix E (continental) or the matrix R (regional) contained information on abiotic variables only. In the third version, the matrix $\mathrm{E}$ (continental) or the matrix $\mathrm{R}$ (regional) contained information on host variables only. In the analyses using only abiotic or only host environment, PCA or PCoA, respectively, was applied as an initial ordination of the matrix E. The main analyses were carried out using the package 'age4' (Chessel et al. 2004, Dray and Dufour 2007, Dray et al. 2007). An example of the annotated $R$ code is presented in Supplementary material Appendix 8. Detailed $\mathrm{R}$ code for the ESLPT analysis can also be found in Pavoine et al. (2011).

\section{Results}

On the continental scale, all eight abiotic environmental variables as well as 18 of 26 host variables demonstrated significant spatial autocorrelation (Supplementary material Appendix 9, Table A9). Therefore, eight host variables (crocidurine shrews, dipodine jerboas, gerbils, harvest mouse, house mouse, mole-rats, rats, steppe lemmings) were omitted from further analyses. Although all six flea traits had significant phylogenetic signal, only four of them (seasonality, abundance, the number of hosts and phylogenetic distinctness of the continental host spectrum) were significantly correlated with environmental variables (Table 1). In addition, flea species that co-occur in a region were significantly more phylogenetically related and significantly more similar in their traits than expected by chance from the continental species pool (i.e. all flea species found in all studied regions) (PQE test: $\beta=0.05$, SES $=4.35$ and TQE test: $\beta=0.05$, $\mathrm{SES}=4.35 ; \mathrm{p}<0.001$ for both).

On the regional scale, most abiotic variables (except for spring NDVI and winter precipitation; Moran's $I=0.21$, $\mathrm{SES}=1.96$ and Moran's $I=0.17, \mathrm{SES}=1.80$, respectively; $\mathrm{p}=0.04$ for both) and all host variables were unaffected by geographic space (Moran's $I=-0.08-0.05$, SES $=0.67-$ 0.95 ; $\mathrm{p}>0.10$ for all). Two flea traits (microhabitat preference and seasonality) carried a significant phylogenetic signal, while no phylogenetic signal was detected for any of the remaining traits (Table 1). The only two traits significantly correlated with environment were abundance and the number of hosts, whereas this was not the case for the remaining traits (Table 1). Furthermore, flea species cooccurring in a locality within Slovakia did not differ phylogenetically from an assemblage of species taken at random from the regional species pool (i.e. all flea species found in Slovakia) (PQE test: $\beta=0.04, \mathrm{SES}=0.36, \mathrm{p}=0.73$ ). The tests of trait clustering were marginally significant suggesting that flea co-occurring in a locality tended to be more similar in their traits than expected by chance (TQE test: $\beta=0.07$, SES $=1.79, p=0.06$ ). Based on these results, we did not include either space or phylogeny in the further analyses on the regional scale and applied regular RLQ analyses. Abundance and the number of hosts exploited were the only flea traits considered in these analyses.

The link between the matrix $\mathrm{R}$ composed of both environmental and spatial variables and the matrix $\mathrm{Q}$ composed of both trait and phylogenetic variables was significant as were the links between the matrices $\mathrm{E}$ and T, $\mathrm{S}$ and T, E and $\mathrm{P}$ and $\mathrm{S}$ and $\mathrm{P}$ (multivariate fourth-corner tests; Table 2).

Table 1. Summary of the tests for phylogenetic signal (PST; root-skewness tests) and correlation with environmental variables (EET; fourth-corner tests) for six flea traits on the continental (C) and the regional (R) scales. OV - observed value, SES - standardized effect size. PHS - phylogenetic host specificity (phylogenetic distinctness of host spectrum). See text and Supplementary material Appendix 3 for trait definitions.

\begin{tabular}{|c|c|c|c|c|c|c|c|}
\hline \multirow[b]{2}{*}{$\underline{\text { Scale }}$} & \multirow[b]{2}{*}{ Trait } & \multicolumn{3}{|c|}{ PST } & \multicolumn{3}{|c|}{ EET } \\
\hline & & OV & SES & p & OV & SES & $\mathrm{p}$ \\
\hline \multirow[t]{6}{*}{$\mathrm{C}$} & Microhabitat preference & 0.12 & -23.85 & $<0.001$ & 0.00006 & -0.26 & 0.49 \\
\hline & Seasonality & 0.15 & -19.55 & $<0.001$ & 0.002 & 4.19 & 0.006 \\
\hline & Abundance & 0.40 & -3.50 & $<0.001$ & 0.0001 & 5.31 & 0.004 \\
\hline & Number of hosts & 0.44 & -2.19 & $<0.01$ & 0.0001 & 9.35 & $<0.001$ \\
\hline & Regional PHS & 0.44 & -2.17 & $<0.01$ & 0.00002 & -0.09 & 0.39 \\
\hline & Continental PHS & 0.45 & -2.65 & $<0.01$ & 0.0001 & 2.00 & 0.04 \\
\hline \multirow[t]{6}{*}{$\mathrm{R}$} & Microhabitat preference & 0.19 & -6.62 & $<0.01$ & 0.0001 & -0.63 & 0.71 \\
\hline & Seasonality & 0.38 & -3.07 & $<0.01$ & 0.0005 & 0.85 & 0.17 \\
\hline & Abundance & 0.42 & -1.34 & 0.10 & 0.0003 & 2.40 & 0.04 \\
\hline & Number of hosts & 0.52 & 0.09 & 0.54 & 0.0001 & 2.50 & 0.04 \\
\hline & Local PHS & 0.62 & 1.31 & 0.92 & 0.00001 & -0.68 & 0.80 \\
\hline & Regional PHS & 0.58 & 0.77 & 0.79 & 0.00002 & -0.62 & 0.69 \\
\hline
\end{tabular}


Table 2. Summary of the forth-corner tests for the association between environmental $(\mathrm{E})$, spatial $(\mathrm{S})$, trait $(\mathrm{T})$ and phylogenetic (P) matrices on the continental scale. OV - observed value, SES standardized effect size (Table 1). See text for definitions of matrices.

\begin{tabular}{lllc}
\hline Pair of matrices & OV & SES & $p$ \\
\hline$R$ and Q & 0.43 & 7.92 & $<0.001$ \\
E and T & 0.0001 & 6.89 & $<0.001$ \\
E and P & 0.01 & 7.21 & $<0.001$ \\
S and T & 0.009 & 5.75 & $<0.001$ \\
S and P & 1.21 & 5.35 & $<0.001$ \\
\hline
\end{tabular}

The first two axes of the ESLTP analysis explained 68.3\% of total variation with the first axis explaining four times more variation than the second axis (54.1 versus $14.2 \%$ ). The abiotic variable with the highest negative correlation with the first axis was summer NDVI/precipitation, whereas maximum altitude had the highest positive correlation (Fig. 1a). The second axis was highly negatively correlated with summer precipitation and highly positively correlated with maximum temperature (Fig. 1a). Regarding host composition variables, the negative areas of the first and the second axes were mainly associated with a) soricine shrews and moles and to a lesser degree with water vole, bank voles and wood mice and b) chipmunk, respectively (Fig. 1b). The positive areas of the first and the second axes were associated with a) allactagine jerboas, small hamsters and mountain voles and b) ground squirrels, allactagine jerboas and marmots, respectively. From the spatial perspective, the coordinates of regions along the first axis reflected north-south changes (Fig. 2a) and the coordinates of regions along the second axis reflected east-west changes (Fig. 2b) in abiotic environment and host composition.
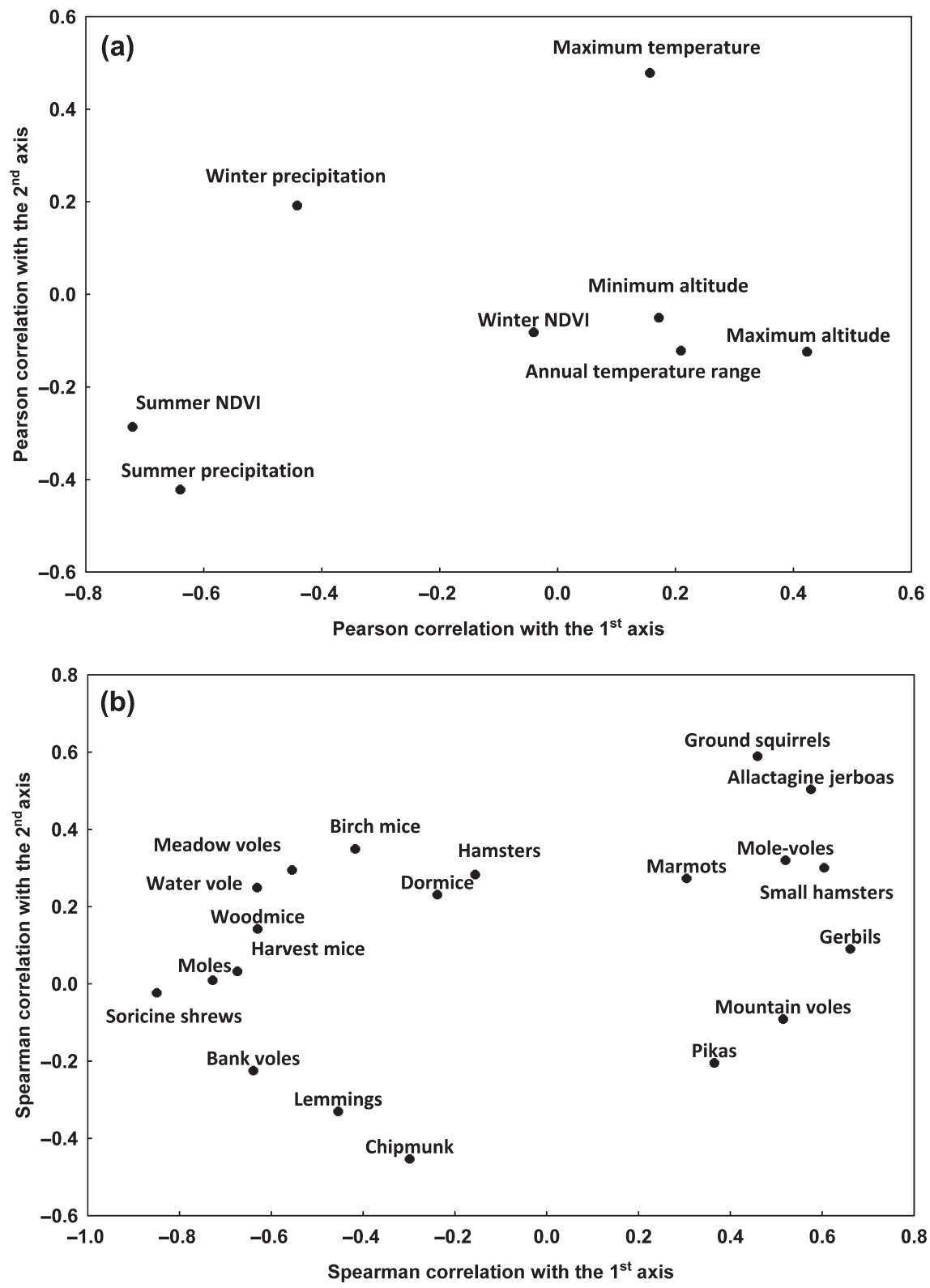

Figure 1. Scatterplots of (a) Pearson correlations between abiotic environmental variables and (b) Spearman correlations between host variables and the coordinates of regions on the 1st and the 2nd axes of the ESLTP analysis (the continental scale). 

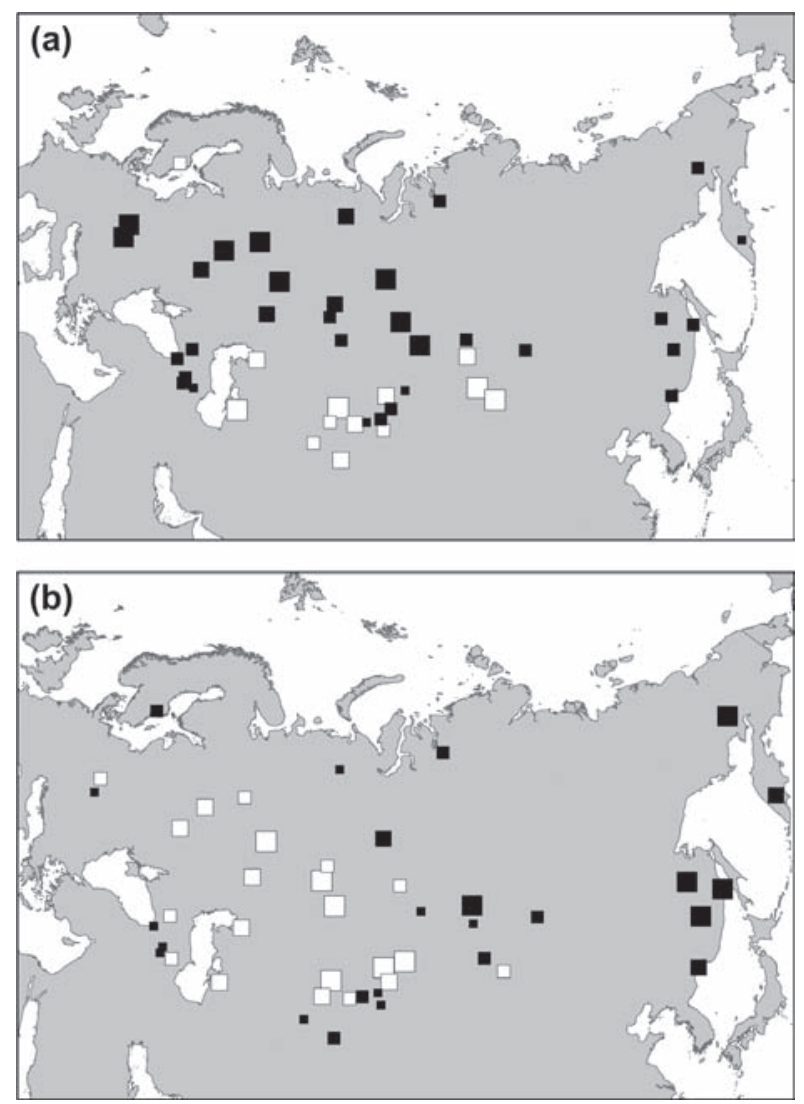

Figure 2. Geographic position and coordinates of regions along the 1 st (a) and the 2nd (b) axes of the ESLTP analysis (the continental scale). White and black squares designate positive and negative coordinates, respectively. The sizes of the squares are proportional to the absolute values of the coordinates.

When the ESLTP analysis was done with only abiotic environmental variables as the matrix $\mathrm{E}$, the proportion of variation explained by the first and the second axes was $58 \%$ (44 and $12 \%$, respectively); that is, substantially lower than that for abiotic and host environment taken together. When the ESLTP analysis was done with only the host environment as the matrix E, the proportion of variation explained by the first and the second axes was slightly higher than when both components of environment were included (54.3 and $14.2 \%$, respectively).

Pearson correlations of flea quantitative traits with the axes of the ESLTP analyses and coordinates of their binary traits on these axes (Supplementary material Appendix 10, Table A10) indicate that fleas in the northern assemblages (negative values along the first axis) were characterized by lower host specificity from both numerical and phylogenetic perspectives (larger and more phylogenetic diverse host spectra), whereas fleas in the southern assemblages (positive values along the first axis) were more host specific (note negative correlation of the number of hosts and phylogenetic distinctness of the host spectrum with the first axis). Furthermore, fleas in the northern assemblages had predominantly summer reproductive periods, whereas fleas in the southern assemblages reproduced either year round or in winter (note negative coordinate of summer reproduction and positive coordinates of winter and yearround reproduction on the first axis). Similarly, many fleas in the eastern assemblages (negative values along the second axis) were abundant, had relatively lower host specificity and reproduced either during the warm season or year round. In contrast, fleas in the western assemblages (positive values along the second axis) were less abundant, more host specific and tended to reproduce during the cold season. These patterns are indicated by positive correlations of the three quantitative traits with as well as negative coordinate of winter reproduction and positive coordinates of winter and year-round reproduction on the second axis (Supplementary material Appendix 10, Table A10).

From the phylogenetic perspective, the southern flea assemblages were dominated by the ceratophyllid genus Nosopsyllus, species belonging to a clade of Frontopsylla, Ophthalmopsylla, and Paradoxopsyllus, and a majority of species belonging to Hystrichopsyllidae II except for Hystrichopsylla (compare Supplementary material Appendix 4, Fig. A4 and Fig. 3). In the northern regions, flea assemblages were represented by ceratophyllids (other than Nosopsyllus), a majority of species from Leptopsyllidae I and species of genera Ctenophthalmus and Hystrichopsylla. Regarding the west-east gradient of flea species composition, most flea lineages could not be explicitly associated with either the eastern or western assemblages. However, representatives of Hystrichopsyllidae II clearly tended to be found in the eastern regions (Supplementary material Appendix 4, Fig. A4 and Fig. 3).

The link between the matrix R (abiotic and host environment) and the matrix Q (flea abundance and number of hosts exploited) was significant (multivariate fourthcorner test; observed value $=0.15, \operatorname{SES}=2.07, \mathrm{p}<0.05$ ) The first axis of the regular RLQ analysis explained $96 \%$ of the total variation. Therefore, we did not include the second axis in the following considerations. The first RLQ axis correlated positively with maximum altitude and summer NDVI and negatively with minimum temperature (Fig. 4a). Regarding host composition, some localities (positive area of the first axis) were associated mainly with dormice and shrews, whereas other localities (negative area of the first axis) were mainly associated with water voles and moles (Fig. 4b). A re-run of the RLQ analyses using abiotic environmental variables only resulted in the first axis explaining more variation than when both abiotic and host environment were used in the analysis (99.8\%). When the analysis was repeated for host variables only (as the matrix R), the proportion of variation explained by the first axis decreased (90.3\%) compared to that from the original RLQ analysis.

Correlations of both flea traits with the first RLQ axis were high and negative $(-0.88$ for abundance and -0.80 for the number of hosts). Consequently, fleas in the localities corresponding to the positive side of the axis (Javorie, Slovensky kras, Kosice, Hylov and Levocske hory) were less abundant and more host specific than fleas in the localities corresponding to the negative side (the remaining eight localities; Fig. 5a). These two types of flea assemblages were characterized by species belonging to different phylogenetic lineages (Fig. 5b). 
Axis 1

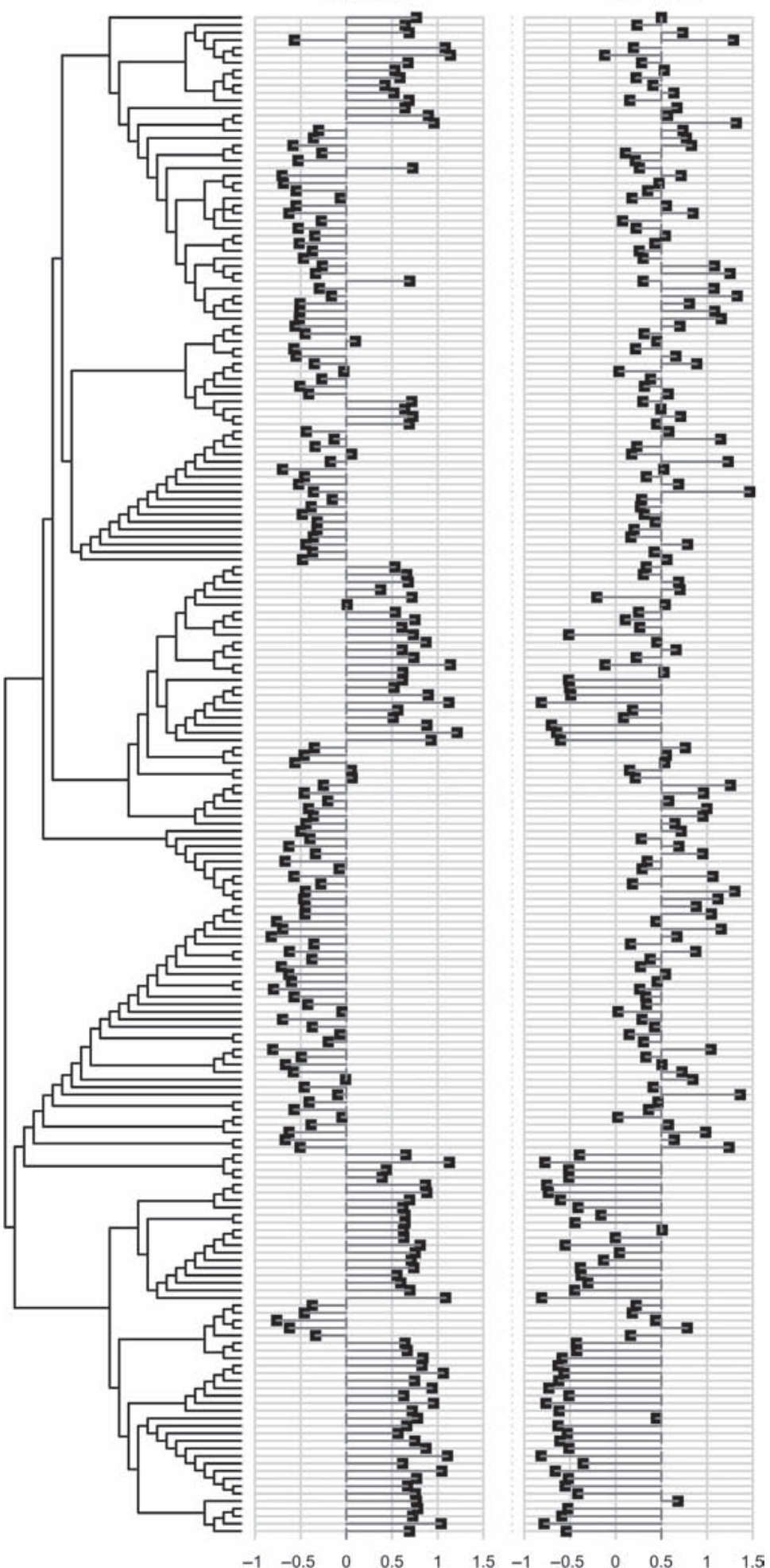

Figure 3. A flea phylogenetic tree and coordinates of flea species on the 1st and the 2nd axes of the ESLTP analysis based on a combination of their traits (the continental scale). See text for details. 


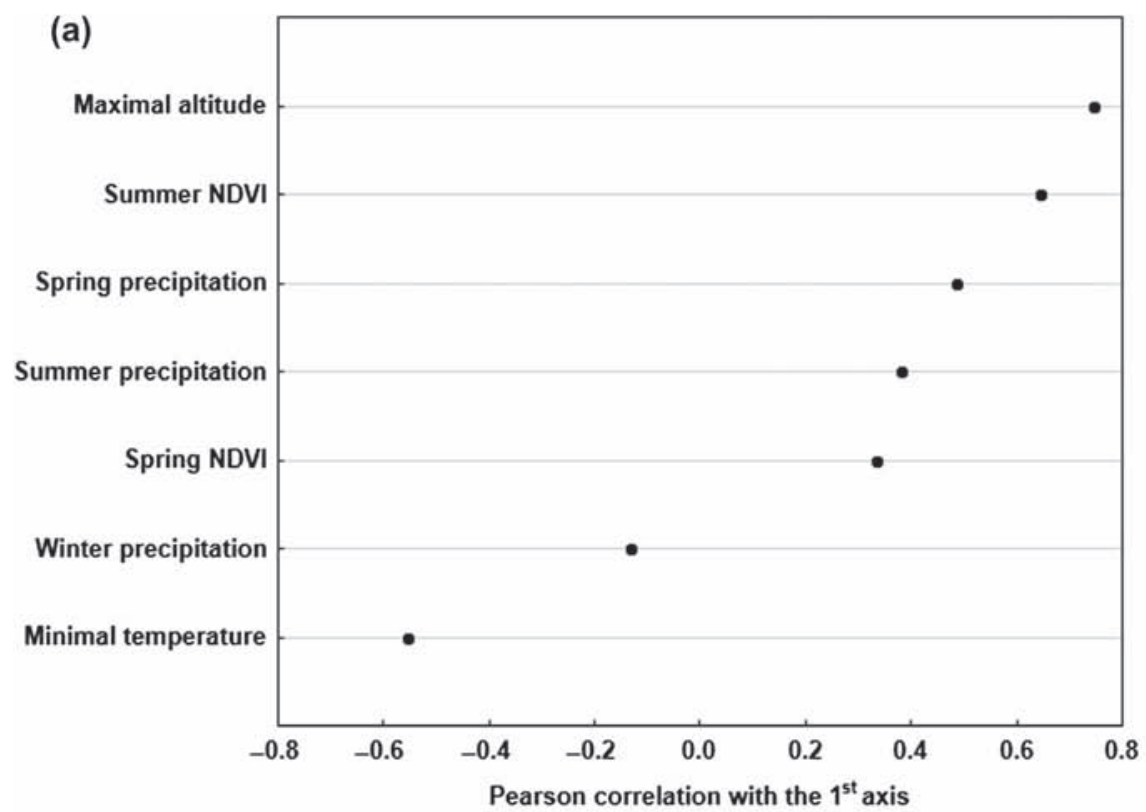

\section{(b)}

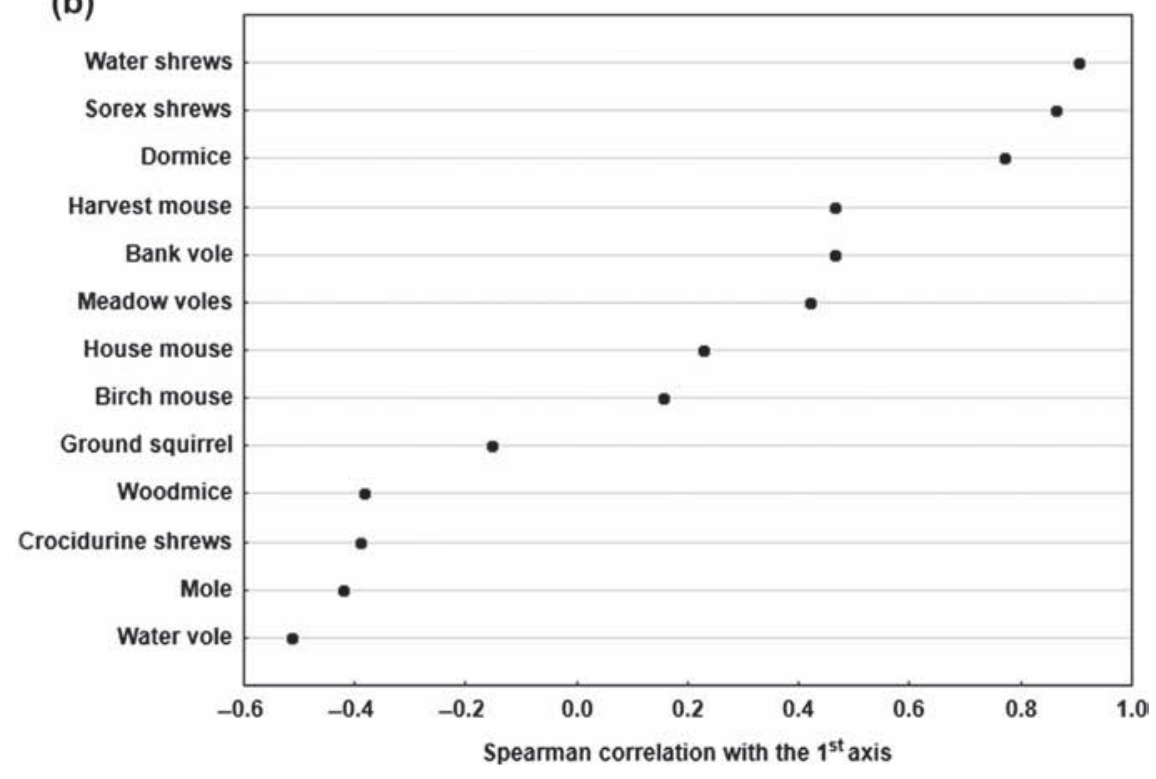

Figure 4. Scatterplots of (a) Pearson correlations between abiotic environmental variables and (b) Spearman correlations between host variables and the coordinates of localities on the 1st axis of the RLQ analysis (the regional scale).

\section{Discussion}

Our results indicate that compound communities of fleas are to a great extent assembled via environmental filters similarly to infra- and component communities of these parasites (Krasnov et al. 2014). Moreover, these environmental filters represent an interplay between filtering through the abiotic environment and filtering through host composition. In other words, abiotic conditions and host composition in a region or a locality act as filters to restrict co-occurring flea species to a certain subset sharing traits (on both continental and regional scale) and phylogenetic affinity (on the continental scale) (Tofts and Silvertown 2000, Statzner et al. 2004; see Krasnov et al. 2014 for fleas). The relative importance of these two components of environmental filtering appears to differ across scales. Host composition had a stronger effect on flea assembly than the abiotic environment on the continental scale, while the opposite was true for the regional scale.

Although the model taxon in our study was represented by parasitic organisms, our main findings may be applied to any biological community. We demonstrated that factors assembling communities of the same taxon may differ substantially between spatial scales and/or hierarchical levels. Furthermore, the effects of geographic space and phylogeny on community assembly appeared to be important on the larger but not on the smaller scale. Further studies linking composition of communities of free-living species with space, environment, traits, and phylogeny are necessary to generalize findings of this study. 


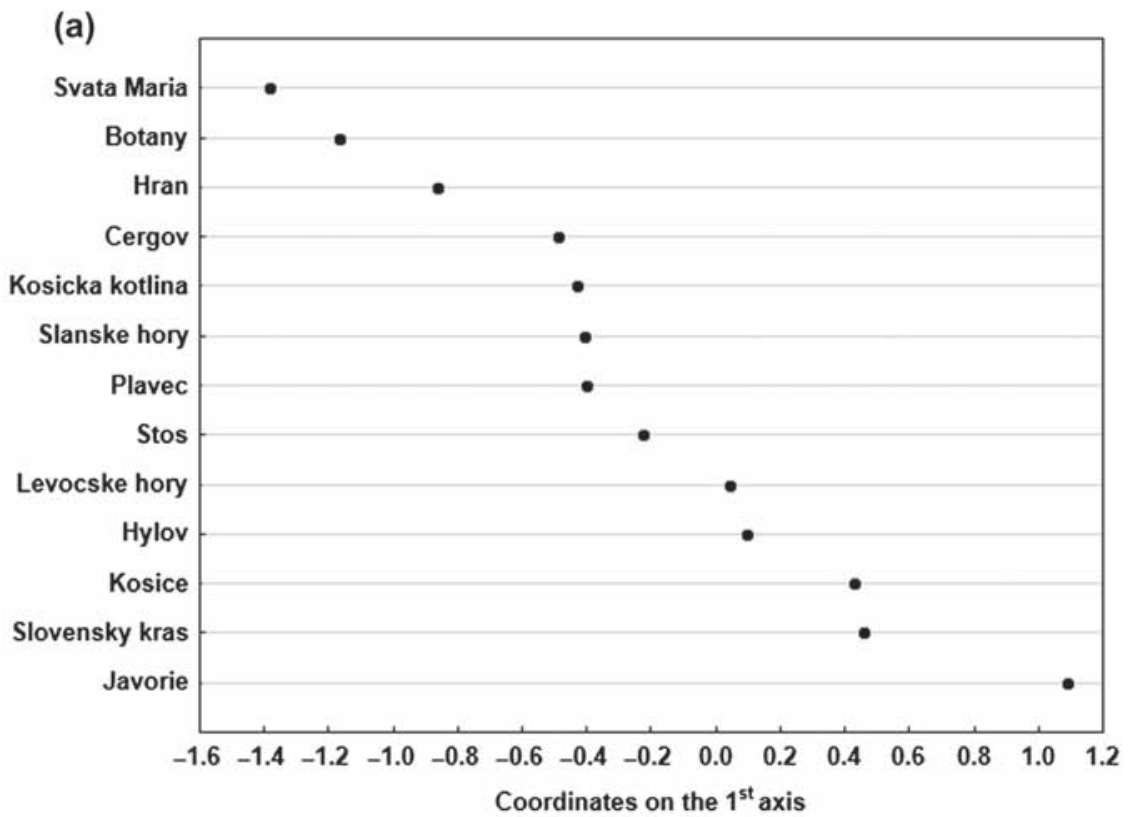

(b)

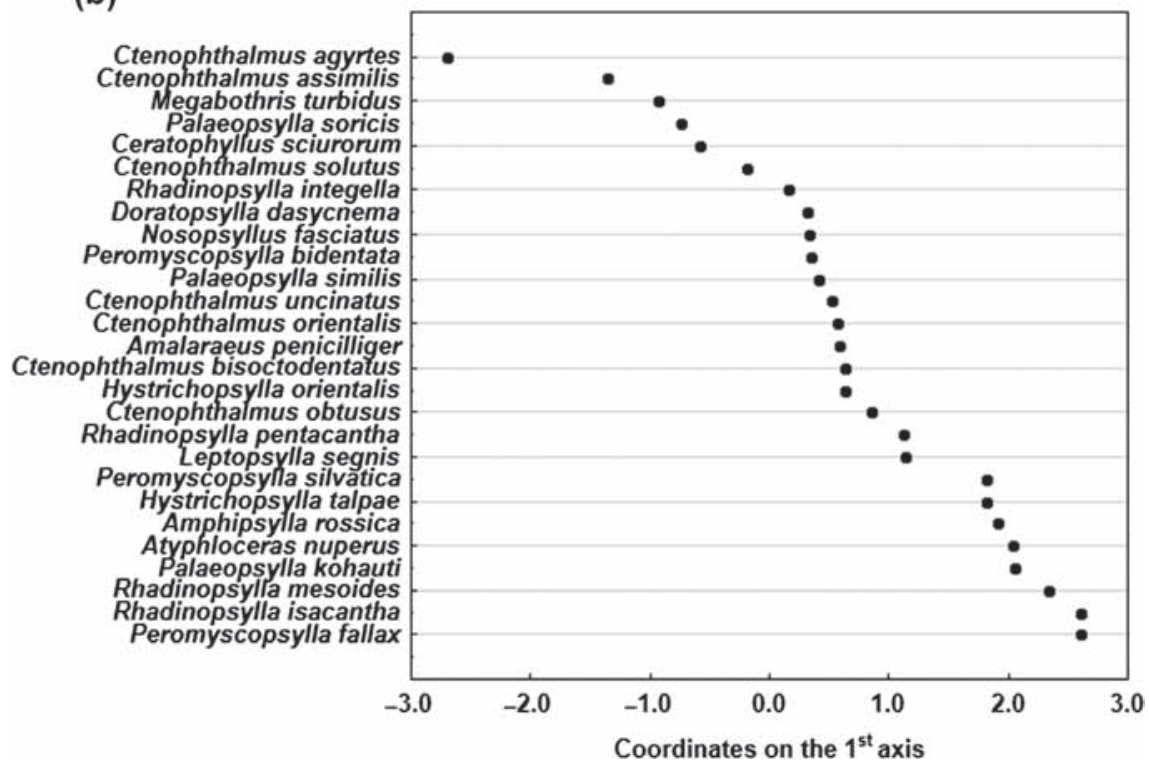

Figure 5. Coordinates of sites (a) and species (b) on the 1st axis of the RLQ analysis (the regional scale).

\section{Abiotic environment, flea traits and flea phylogeny}

The effect of abiotic environmental variables on adult and immature fleas has been repeatedly demonstrated (reviewed by Krasnov 2008). In particular, air temperature and relative humidity in the hosts' burrows, which obviously correlate with precipitation, altitude and amount of green vegetation, strongly influence egg production and rate of oviposition (Kunitsky 1961), development and survival of preimaginal fleas (Krasnov et al. 2001a, b) and survival of imagoes (Krasnov et al. 2002b). The mechanisms behind this strong microclimatic effect on flea performance are purely physiological. For example, larval fleas cannot close their spiracles, so they are extremely sensitive to low humidity (Mellanby 1933).

Different flea species often demonstrate different preferences for air temperature and humidity (Krasnov et al. 2001a). These species-specific microclimatic preferences may be echoed in other flea traits such as seasonality. For example, Frontopsylla semura, which exhibits a decrease in the rate of oviposition with increasing air temperature is characterized by winter periods of activity and breeding (Bryukhanova and Myalkovskaya 1974). Furthermore, microclimatic preferences and patterns of seasonality seem to be phylogenetically constrained to a certain extent. For example, all species of the genus Stenoponia occur as imagoes and reproduce in the cold seasons, independent of climate of the region that they inhabit (Vashchenok 1988, Krasnov 2008). Among pulicid fleas, no species is active only in the cold season, despite the ubiquitous distribution of this family (Krasnov 2008). However, other genera (e.g. Nosopsyllus) present no evidence of phylogenetically constrained seasonality and demonstrate a wide variety of seasonal patterns (Krasnov et al. 2002a). 
In addition to the direct effects of abiotic environment on flea assemblages, it may act indirectly via its effect on host assemblages. Environment may operate as a filter, so that a host community will consist from phylogenetically closely-related species that are ecologically (e.g. in structure of their shelters) and physiologically (e.g. in the pattern of their immune response) similar. However, phylogenetic relatedness is not a necessary condition for the effect of abiotic environment on parasites mediated via hosts. Abiotic environment may require even phylogenetically distant cooccurring hosts to share certain adaptations. For example, rainfall and soil structure may affect burrowing habits of multiple small mammal species resulting in among-species similarity in depth, ventilation, temperature and /or humidity of the burrow environment which, in turn, may affect assembly of flea communities. Mediation of the environmental effects on parasite assemblages by its effects on host assemblages warrants further investigation. Although we did not find correlation between abiotic environment and host species composition in our study, the difference between scales in relative importance of abiotic versus host environment suggests that the possible role of host assemblages as a mediator between abiotic environment and parasite assemblages will likely be more pronounced on larger spatial scales.

\section{Host composition, flea phylogeny and flea traits}

The association between host composition, in terms of species and phylogenetic lineages, and species and phylogenetic composition of flea assemblages found in this study is not surprising because hosts are of the utmost importance for parasites. Krasnov et al. (2012a) suggested that the species composition of flea assemblages depends strongly on the species composition of their host assemblages but is only weakly related to host phylogeny. Indeed, a parasite may occur in a region if it either originated there or if it arrived from elsewhere. In either of these cases, it may switch to a different host if its original host, whether it was autochthonous or invading, became extinct. Experimental evidence shows that a parasite may rapidly acquire adaptations to exploit successfully a new host and concomitantly lose the ability to exploit a previous host (see Arbiv et al. 2012 for fleas).

Results of the present study suggest that the identities of host phylogenetic lineages may, nevertheless, act as an important filter in the process of assembling compound communities of fleas. There is no doubt that the origin and diversification of fleas are associated with diversification of their mammalian hosts (Whiting et al. 2008). Although strict co-speciation between fleas and mammals has not been found and host-switching (Paterson and Banks 2001) has seemed to be the main type of event in the evolutionary history of their associations (Krasnov and Shenbrot 2002, Lu and Wu 2005), available evidence suggests that diversification of at least some higher flea taxa took place within certain taxa of their mammalian hosts (Traub 1985, Whiting et al. 2008). For example, evolution of the genera Hystrichopsylla and Mesopsylla is thought to be associated mainly with soricomorph and dipodid hosts, respectively (Traub 1985, Lewis and Eckerlin 2004).

We found that host filters limit regional or local flea assemblages to a subset of species characterized by a certain level of abundance and host specificity. On the continental scale, this can be explained by the fact that these traits are phylogenetically constrained (Krasnov et al. 2011b), so that the filtering of flea phylogenetic lineages will inevitably lead to the filtering of flea traits. Another mechanism that can act on both scales is related to the fact that both these traits are species-specific characters not only of a flea species, but to a lesser extent of a host species (Krasnov et al. 2004, 2006b). In other words, fleas found on a certain host species or a host taxon are often characterized by similar abundance and/or host specificity. For example, some host properties may constrain the number of fleas harbored by an individual. The abundance of imago fleas may be limited by the level of the host's species-specific immune (Klein and Nelson 1998, Goüy de Bellocq et al. 2006) and/or behavioral (Mooring et al. 2000) defenses, while the abundance of immature fleas is likely limited by the host sheltering behavior and its burrow or nest structure (Krasnov et al. 2001a).

\section{Spatial effects}

Ecological communities of living species are not isolated entities; rather they are composed of populations that are subjected to colonization and extinction dynamics, which vary across spatiotemporal scales (Chase and Leibold 2003). It is therefore important to analyze community structure as a function of dispersal as proposed $50 \mathrm{yr}$ ago by Preston (1962a, b) and by MacArthur and Wilson $(1963,1967)$ in the context of a dynamic equilibrium. Space shapes the level of dissimilarity between communities in the sense that geographic distance tends to decrease the number of common species, a pattern early labelled as the distance decay of similarity (Nekola and White 1999). The effect of geographic space on flea compound communities found in the present study on the continental scale supports the results of earlier studies that showed the occurrence of distance decay of similarity in communities of various parasites (including fleas) on various hierarchical scales (Poulin 2003, Krasnov et al. 2010).

Another spatial effect found in our study is that fleas from the southern assemblages had, on average, higher host specificity than fleas from the northern assemblages. This finding is compatible with the predictions of recent hypotheses about latitudinal gradients in niche breadth (reviewed by Vázquez and Stevens 2004) and supports earlier results for both freeliving (Hernández Fernández and Vrba 2005, Kubota et al. 2007) and parasitic organisms (Krasnov et al. 2008). This pattern may arise because a) specialized species can only tolerate restricted abiotic and biotic conditions (Brown 1995) and b) the level of specialization is determined by population stability which, in turn, is determined by environmental stability (MacArthur 1955, 1972). Lower stability and higher seasonality in northern regions compared to southern regions may thus produce negative specialization-latitude relationships (MacArthur 1972, Vázquez and Stevens 2004).

\section{Scale-dependent variation in the relative importance of host and abiotic filters}

Assembly rules appeared to differ between regional and local flea communities. The most intriguing discovery of 
the present study is that relative roles of abiotic and host filters differ dramatically between scales. Variation in species composition of flea communities across regions within a continent was better explained by host species composition than by abiotic environment, while the opposite was true for local communities within a region. In addition, in contrast to regional communities, species composition of local communities was not affected by either geographic space or flea phylogeny. This supports earlier reports of scale-dependence of community processes that was found for host-parasite associations other than those between fleas and mammals (Poulin and Valtonen 2001) and for other than species assembly patterns (Krasnov et al. 2011c).

On both scales, parasite community composition is a net result of colonization and extinction of parasites (Poulin 2007). However, the mechanisms of colonization and extinction dynamics might be profoundly different at different scales. Across localities within a region, colonizations and extinctions are mainly governed by epidemiological processes via differential birth and death dynamics (Morand et al. 2002). Moreover, similarity in host composition across localities within a region is much higher than that across regions within a continent (pairwise Bray-Curtis similarity ranging from 42.1 to 90.0 and being 66.8 on average versus pairwise Bray-Curtis similarity ranging from 0 to 74.10 and being 38.2 on average, respectively). As a result, in different locations the same host species often harbor different flea species. For example, flea assemblages of Sorex araneus differ between lowland and montane habitats due to the occurrence of Palaeopsylla soricis mainly in the former and Palaeopsylla similis mainly in the latter (see other examples with fleas of Slovakia in Krasnov et al. 2006b). A similar pattern in other host and flea species has been reported for other regions and explained by the different preferences of fleas for abiotic environment (Krasnov et al. 1997).

In contrast, across regions within a continent, the dynamics of colonization and extinction of parasites is influenced mainly by evolutionary, biogeographic and historical forces related to their hosts (Hoberg and Brooks 2008). For example, the composition of regional parasite assemblages may be a result of a parasite's response to the 'taxon pulses' of the entire biota to which they belong. The concept of 'taxon pulses' posits that the composition of a biota is determined by the alternation of periods of isolation (producing stable and endemic ecological associations) with periods of expansion (when members of different ecological associations may contact) (Hoberg and Brooks 2008). The co-diversification of parasites with their hosts might be a predominant process shaping parasite assemblages during isolation phases, while colonization of new hosts might be predominant during expansion phases (Hoberg and Brooks 2008). Hostswitching is not precluded during isolation phases but it does not affect species composition of a regional parasite community. Obviously, both processes operating on parasites are tightly associated with their hosts which are affected by taxon pulses as well. As a result, hosts act as the most important filter assembling regional parasite communities on the continental scale.

In conclusion, our study demonstrates that the key factors underlying structure of compound flea communities are scale-dependent. Assembly rules on the continental scale are influenced by geographic space, abiotic environment, host composition and flea phylogeny with the effect of host composition being more important than that of the abiotic environment. In contrast, on the regional scale, geographic space and flea phylogeny do not play any significant role, while the effect of abiotic environment appeared to be more important than that of host composition. These results together with the results of our earlier studies of factors assembling flea communities (Krasnov et al. 2014) suggest that these communities are governed by environmental (both biotic and abiotic) filters rather than by competitive species interactions. Furthermore, the approach used in this study demonstrated a substantial phylogenetic component in the assembly of compound communities of fleas on the continental scale, whereas our earlier studies (Krasnov et al. 2012b) failed to reveal this.

Acknowledgements - We thank Sandrine Pavoine for help with the $\mathrm{R}$ code. We thank Berry Pinshow for helpful comments on the earlier version of the manuscript. Studies in Slovakia were done under the licenses of the Ministry of Environment of the Slovak Republic no. 297/108/06-3.1 and no. 6743/2008-2.1. This study was partly supported by grants from VEGA (1/0390/12 to MS) and the Israel Science Foundation (grant no. $26 / 12$ to BRK and ISK). This is publication number 838 of the Mitrani Dept of Desert Ecology.

\section{References}

Ackerly, D. D. and Cornwell, W. K. 2007. A trait-based approach to community assembly: partitioning of species trait values into within- and among-community components. - Ecol. Lett. 10: $135-145$.

Arbiv, A. et al. 2012. Use it or lose it: reproductive implications of experimental host shifting in a haematophagous ectoparasite. - J. Evol. Biol. 25: 1140-1148.

Brown, J. H. 1995. Macroecology. - Univ. Chicago Press.

Bryukhanova, L. V. and Myalkovskaya, C. A. 1974. On the duration of metamorphosis of fleas in the nests of the pygmy ground squirrel. - In: Pilipenko, V. G. (ed.), Particularly dangerous diseases in Caucasus. Proceedings of III ScientificPractical Conference of the Anti-Plague Establishments of Caucasus on Natural Focality, Epidemiology and Prophylaxis of Particularly Dangerous Diseases 14-16 May 1974. Sci. AntiPlague Inst. Caucasus and Trans-Caucasus, pp. 121-124, in Russian.

Cavender-Bares, J. et al. 2004. Phylogenetic overdispersion in Floridian oak communities. - Am. Nat. 163: 823-843.

Cavender-Bares, J. et al. 2009. The merging of community ecology and phylogenetic biology. - Ecol. Lett. 12: 693-715.

Chase, J. M. and Leibold, M. A. 2003. Ecological niches: linking classical and contemporary approaches. - Univ. Chicago Press.

Chessel, D. et al. 2004. The ade4 package - I: one-table methods. - R News 4: 5-10.

Chesson, P. 2000. Mechanisms of maintenance of species diversity. - Annu. Rev. Ecol. Syst. 31: 343-366.

Darwin, C. R. 1859. On the origin of species by means of natural selection, or the preservation of favoured races in the struggle for life. - John Murray, London.

Dolédec, S. et al. 1996. Matching species traits to environmental variables: a new three-table ordination method. - Environ. Ecol. Stat. 3: 143-166.

Dray, S. and Dufour, A. B. 2007. The ade4 package: implementing the duality diagram for ecologists. - J. Stat. Softw. 22: 1-20. 
Dray, S. and Legendre, P. 2008. Testing the species traitsenvironment relationships: the fourth-corner problem revisited. - Ecology 89: 3400-3412.

Dray, S. et al. 2003. Co-inertia analysis and the linking of ecological data tables. - Ecology 84: 3078-3089.

Dray, S. et al. 2007. The ade4 package - II: two-table and K-table methods. - R News 7: 47-52.

González, M. T. and Oliva, M. E. 2009. Is the nestedness of metazoan parasite assemblages of marine fishes from the southeastern Pacific coast a pattern associated with the geographical distributional range of the host? - Parasitology 136: 401-409.

Goüy de Bellocq, J. et al. 2006. Temporal dynamics of a T-cell mediated immune response in desert rodents. - Comp. Biochem. Physiol. A 145: 554-559.

Hardy, O. J. and Senterre, B. 2007. Characterizing the phylogenetic structure of communities by an additive partitioning of phylogenetic diversity. - J. Ecol. 95: 493-506.

Hernández Fernández, M. and Vrba, E. S. 2005. Rapoport effect and biomic specialization in African mammals: revisiting the climatic variability hypothesis. - J. Biogeogr. 32: 903-918.

Hijmans, R. J. et al. 2005. Very high resolution interpolated climate surfaces for global land areas. - Int. J. Climatol. 25: 1965-1978.

Hoberg, E. P. and Brooks, D. R. 2008. A macroevolutionary mosaic: episodic host-switching, geographical colonization and diversification in complex host-parasite systems. - Ecol. Lett. 35: 1533-1550.

Holmes, J. C. and Price, P. W. 1986. Communities of parasites. - In: Kittawa, J. and Anderson, D. J. (eds), Community ecology: pattern and process. Blackwell, pp. 187-213.

Hubbell, S. P. 2001. The unified neutral theory of biodiversity and biogeography. - Princeton Univ. Press.

Hutchinson, G. E. 1959. Homage to Santa Rosalia, or why are there so many kinds of animals? - Am. Nat. 93: 145-159.

Ingram, T. and Shurin, J. B. 2009. Trait-based assembly and phylogenetic structure in northeast Pacific rockfish assemblages. - Ecology 90: 2444-2453.

Khokhlova, I. S. et al. 2012. Ectoparasite fitness in auxiliary hosts: phylogenetic distance from a principal host matters. - J. Evol. Biol. 25: 2005-2013.

Kingston, T. et al. 2000. Resource partitioning in rhinolophoid bats revisited. - Oecologia 124: 332-342.

Klein, S. L. and Nelson, R. J. 1998. Adaptive immune responses are linked to the mating system of arvicoline rodents. - Am. Nat. 151: 59-67.

Krasnov, B. R. 2008. Functional and evolutionary ecology of fleas. A model for ecological parasitology. - Cambridge Univ. Press.

Krasnov, B. R. and Shenbrot, G. I. 2002. Coevolutionary events in history of association of jerboas (Rodentia: Dipodidae) and their flea parasites. - Isr. J. Zool. 48: 331-350.

Krasnov, B. R. et al. 1997. Host-habitat relation as an important determinant of spatial distribution of flea assemblages (Siphonaptera) on rodents in the Negev Desert. - Parasitology 114: 159-173.

Krasnov, B. R. et al. 2001a. The effect of air temperature and humidity on the survival of pre-imaginal stages of two flea species (Siphonaptera: Pulicidae). - J. Med. Entomol. 38: 629-637.

Krasnov, B. R. et al. 2001b. Development rates of two Xenopsylla flea species in relation to air temperature and humidity. - Med. Vet. Entomol. 15: 249-258.

Krasnov, B. R. et al. 2002a. Annual cycles of four flea species (Siphonaptera) in the central Negev desert. - Med. Vet. Entomol. 16: 266-276.

Krasnov, B. R. et al. 2002b. Time to survival under starvation in two flea species (Siphonaptera: Pulicidae) at different air temperatures and relative humidities. - J. Vector Ecol. 27: $70-81$.

Krasnov, B. R. et al. 2004. Geographical variation in host specificity of fleas (Siphonaptera): the influence of phylogeny and local environmental conditions. - Ecography 27: 787-797.

Krasnov, B. R. et al. 2005. Nested pattern in flea assemblages across the host's geographic range. - Ecography 28: 475-484.

Krasnov, B. R. et al. 2006a. Are ectoparasite communities structured? Species co-occurrence, temporal variation and null models. - J. Anim. Ecol. 75: 1330-1339.

Krasnov, B. R. et al. 2006b. Habitat variation in species composition of flea assemblages on small mammals in central Europe. - Ecol. Res. 21: 460-469.

Krasnov, B. R. et al. 2008. Latitudinal gradients in niche breadth: empirical evidence from haematophagous ectoparasites. - J. Biogeogr. 35: 592-601.

Krasnov, B. R. et al. 2010. Similarity in ectoparasite faunas of Palaearctic rodents as a function of host phylogenetic, geographic, or environmental distances: which matters the most? - Int. J. Parasitol. 40: 807-817.

Krasnov, B. R. et al. 2011a. Aggregative structure is the rule in communities of fleas: null model analysis. - Ecography 34: 751-761

Krasnov, B. R. et al. 2011b. Scale-dependence of phylogenetic signal in ecological traits of ectoparasites. - Ecography 34: $114-122$.

Krasnov, B. R. et al. 2011c. Nestedness and beta-diversity in ectoparasite assemblages of small mammalian hosts: effects of parasite affinity, host biology and scale. - Oikos 120: 630-639.

Krasnov, B. R. et al. 2012a. Compositional and phylogenetic dissimilarity of host communities drives compositional and phylogenetic dissimilarity of ectoparasite assemblages: geographic variation and scale-dependence. - Parasitology 139: 338-347.

Krasnov, B. R. et al. 2012b. Phylogenetic signal and species roles in compartmentalized mammal-flea networks. - Am. Nat. 179: 501-511.

Krasnov, B. R. et al. 2014. Co-occurrence and phylogenetic distance in communities of mammalian ectoparasites: limiting similarity versus environmental filtering. - Oikos 123: 63-70.

Kubota, U. et al. 2007. Body size and host range co-determine the altitudinal distribution of Neotropical tephritid flies. - Global Ecol. Biogeogr. 16: 632-639.

Kunitsky, V. N. 1961. On the environmental conditions of fleas parasitic on gerbils in southeastern Azerbaijan SSR. - Zool. Zhurnal 40: 848-858.

Lewis, R. E. and Eckerlin, R. P. 2004. A new species of Hystrichopsylla Taschenberg, 1880 (Siphonaptera: Hystrichopsyllidae) from Guatemala. - Proc. Entomol. Soc. Wash. 106: 757-760.

Lu, L. and Wu, H. 2005. Morphological phylogeny of Geusibia Jordan, 1932 (Siphonaptera: Leptopsyllidae) and the hostparasite relationships with pikas. - Syst. Parasitol. 61: 65-78.

MacArthur, R. H. 1955. Fluctuations of animal populations and a measure of community stability. - Ecology 36: 533-536.

MacArthur, R. H. 1972. Geographical ecology. - Princeton Univ. Press.

MacArthur, R. H. and Wilson, E. O. 1963. An equilibrium theory of insular zoogeography. - Evolution 17: 373-387.

MacArthur, R. H. and Levins, R. 1967. The limiting similarity, convergence, and divergence of coexisting species. - Am. Nat. 101: 377-385.

MacArthur, R. H. and Wilson, E. O. 1967. The theory of island biogeography. - Princeton Univ. Press.

Maddison, W. P. and Maddison, D. R. 2011. Mesquite: a modular system for evolutionary analysis, version 2.75. - < http:// mesquiteproject.org $>$. 
Mellanby, K. 1933. The influence of the temperature and humidity on the pupation of Xenopsylla cheopis. - Bull. Entomol. Res. 24: 197-202.

Mooring, M. S. et al. 2000. Testing the interspecific body size principle in ungulates: the smaller they come, the harder they groom. - Anim. Behav. 60: 35-45.

Morand, S. et al. 2002. Order in ectoparasite communities of marine fish is explained by epidemiological processes. - Parasitology 124: S57-S63.

Mouillot, D. et al. 2006. Conservatism of host specificity in parasites. - Ecography 29: 596-602.

Nekola, J. C. and White, P. S. 1999. The distance decay of similarity in biogeography and ecology. - J. Biogeogr. 26: 867-878.

Paterson, A. M. and Banks, J. 2001. Analytical approaches to measuring cospeciation of host and parasites; through a glass, darkly. - Int. J. Parasitol. 31: 1012-1022.

Pavoine, S. et al. 2009. Hierarchical partitioning of evolutionary and ecological patterns in the organization of phylogenetically structured species assemblages: application to rockfish (genus: Sebastes) in the Southern California Bight. - Ecol. Lett. 12: 898-908.

Pavoine, S. et al. 2010. Decomposition of trait diversity among the nodes of a phylogenetic tree. - Ecol. Monogr. 80: 485-507.

Pavoine, S. et al. 2011. Linking patterns in phylogeny, traits, abiotic variables and space: a novel approach to linking environmental filtering and plant community assembly. - J. Ecol. 99: 165-175.

Pettorelli, N. et al. 2005. Using the satellite-derived NDVI to assess ecological effects of environmental change. - Trends Ecol. Evol. 20: 503-510.

Poulin, R. 2003. The decay of similarity with geographical distance in parasite communities of vertebrate hosts. - J. Biogeogr. 30: $1609-1615$.

Poulin, R. 2007. Evolutionary ecology of parasites: from individuals to communities, 2nd ed. - Princeton Univ. Press.

Poulin, R. and Valtonen, E. T. 2001. Nested assemblages resulting from host-size variation: the case of endoparasite communities in fish hosts. - Int. J. Parasitol. 31: 1194-1204.

Preston, F. W. 1962a. The canonical distribution of commonness and rarity. Part 1. - Ecology 43: 185-215.

Preston, F. W. 1962b. The canonical distribution of commonness and rarity. Part 2. - Ecology 43: 410-432.

Purves, D. W. and Pacala, S. W. 2005. Ecological drift in nichestructured communities: neutral pattern does not imply neutral process. - In: Burslem, D. et al. (eds), Biotic interactions in the tropics. Cambridge Univ. Press, pp. 107-138.

Rao, C. R. 1982. Diversity and dissimilarity coefficients: a unified approach. - Theor. Popul. Biol. 21: 24-43.

Schluter, D. 2000. Ecological character displacement in adaptive radiation. - Am. Nat. 156: S4-S16.

Schoener, T. W. 1974. Resource partitioning in ecological communities. - Science 185: 27-39.

Šimková, A. et al. 2001. Order and disorder in ectoparasite communities: the case of congeneric gill monogeneans (Dactylogyrus spp.). - Int. J. Parasitol. 31: 1205-1210.

Stanko, M. 1994. Fleas synusy (Siphonaptera) of small mammals from the central part of the east-Slovakian lowlands. - Biologia (Bratislava) 49: 239-246.

Statzner, B. et al. 2004. Biological trait composition of European stream invertebrate communities: assessing the effects of various trait filter types. - Ecography 27: 470-488.

Thioulouse, J. et al. 1995. Multivariate analysis of spatial patterns: a unified approach to local and global structures. - Environ. Ecol. Stat. 2: 1-14.

Tofts, R. and Silvertown, J. 2000. A phylogenetic approach to community assembly from a local species pool. - Proc. R. Soc. B 267: 363-369.

Traub, R. 1985. Coevolution of fleas and mammals. - In: Kim, K. C. (ed.), Coevolution of parasitic arthropods and mammals. Wiley, pp. 295-437.

Vashchenok, V. S. 1988. Fleas - vectors of pathogens causing diseases in humans and animals. - Nauka, in Russian.

Vázquez, D. and Stevens, R. D. 2004. The latitudinal gradient in niche breadth: concepts and evidence. - Am. Nat. 164: E1-E19.

Vergnon, R. et al. 2013. Repeated parallel evolution reveals limiting similarity in subterranean diving beetles. - Am. Nat. 182: $67-75$.

Violle, C. et al. 2011. Phylogenetic limiting similarity and competitive exclusion. - Ecol. Lett. 14: 782-787.

Webb, C. O. et al. 2002. Phylogenies and community ecology. - Annu. Rev. Ecol. Syst. 33: 475-505.

Weiher, E. et al. 2011. Advances, challenges and a developing synthesis of community ecology theory. - Phil. Trans. R. Soc. B 366: 2403-2413.

Whiting, M. F. et al. 2008. A molecular phylogeny of fleas (Insecta: Siphonaptera): origins and host associations. - Cladistics 24: 677-707.

Supplementary material (Appendix ECOG-00915 at $<$ www.ecography.org/readers/appendix $>$ ). Appendix 1-10. 